\title{
Systems Thinking as an Emergent Team Property: Ongoing research into the enablers and barriers to team-level systems thinking
}

\author{
Caroline Twomey Lamb and Donna H. Rhodes \\ Massachusetts Institute of Technology \\ Building 41-205, 77 Massachusetts Avenue \\ Cambridge, MA 02139 \\ Phone: 617-308-0954, Fax: 617-258-7845 Email: cmtwomey@mit.edu
}

\begin{abstract}
This paper describes ongoing research exploring systems thinking at the team level. Termed collaborative systems thinking by the authors, the concept of higher level systems thinking is envisioned as a means both to build workforce competency and to explicitly deal with system complexity at a higher level within an organization. This paper introduces the key research questions, an initial definition of collaborative systems thinking, demographic and technical motivators, and summarizes the research progress to date and plan for completion. The results of this research will inform the design of technical processes and provide empirical knowledge to support workforce development interventions aimed at developing systems thinking within engineering teams. The role of organizational culture is also considered as a factor in enabling collaborative systems thinking.
\end{abstract}

Keywords - systems thinking, collaborative systems thinking, standard technical process, organizational culture.

\section{INTRODUCTION}

Engineered systems are increasingly complex. Complexity is introduced through advancements in technology and through the logistics required to design and field these systems. As complexity increases, an ever smaller fraction of the design knowledge is documented [1]. For a basic system component, $85 \%$ of the design knowledge is documented. By contrast, only $30 \%$ of a simple system's design knowledge is documented [1]. The remaining $70 \%$ is tacit knowledge encapsulated in the experiences of the designers. The ability to recall and apply this knowledge to solve design problems is an application of systems thinking, a skill recognized as critical to problem solving [2]. As the complexity of systems increases, systems thinking become more important as a means to solve and avoid design problems. However, as complexity increases, the base of knowledge and experience required to solve design problems also grows. This increased requirement for both breadth and depth of experience drives the move towards exploring systems thinking as a team-based property. This next step in the 'hierarchy of systems thinking' will enable complexity to be explicitly addressed at a higher level [3].

Discussions with practicing engineers illustrate examples of teams that 'clicked' and others that missed the mark. While many factors contribute to a team's success of failure, the literature points to several culture and process based factors which the authors see as linked to systems thinking. For example, successful teams engage in extensive problem space exploration before proposing and evaluating alternative [4]. Anecdotal evidence points to these successful teams as system-centric rather than process centric. That is, these teams focused on, and were motivated by, the system under design rather than the process of design. These teams engage in meaningful information exchanges that ensure members have sufficient information to make good decisions [5].

When considering systems of systems, the knowledge and experience required to make decisions crosses systems boundaries, multiple corporate cultures, and several sets of process. As defined by [6], systems of systems realize additional value or functionality by combining constituent systems. These systems of systems are characterized by operational and managerial independence [7]. Lacking a central authority to make decisions, systems of systems' management must draw upon the tacit knowledge base of each system in order to realize the greatest possible value. Systems thinking in this context will be influenced by the varied organizational cultures, processes, and values (e.g. protecting proprietary information). These influences are barriers to the free information exchange and system-centric perspectives shown effective in literature and practice. A better understanding of the key cultural and technical process enablers of higher-level systems thinking would provide system of systems teams with a framework around which to structure their system of systems enterprises and information and decision making processes to promote systems thinking and systems awareness.

This research focuses on understanding team-level systems thinking, or collaborative systems thinking, as a precursor to organizational and systems or system-level systems thinking. In the context of single-system design, multiple disciplines and components must be brought together. Traditional practices place an individual or small group of individuals in charge of managing system-level issues. These systems engineers use tools and processes to consider the implications of design decisions and guide the team through the design process [8]. Systems thinking capability greatly enhances the performance of these systems engineers [9]. Collaborative systems thinking seeks to identify the conditions under which teams are able to think 
systematically and therefore work more efficiently within the systems engineering framework.

The outcomes of this research will influence process design and workforce development in organizations seeking to develop systems thinking. Understanding collaborative systems thinking builds upon past research on individual systems thinking and will enable future research on organizational systems thinking and inter-organizational systems thinking in support of systems of systems.

\section{SYSTEMS THINKING}

Systems thinking is a term used and defined in several different contexts. Most popular definitions originate in the field of systems dynamics [10,11, 12, 13, and 14] and refer to well defined bodies of knowledge and tool sets [13]. Figure 1 shows a sampling of these definitions. Though different in wording, the common themes of complexity, interrelationships, context dependency, emergent behavior, and wholism repeat throughout the definitions and are visually represented by the vertical arrows in Figure 1.

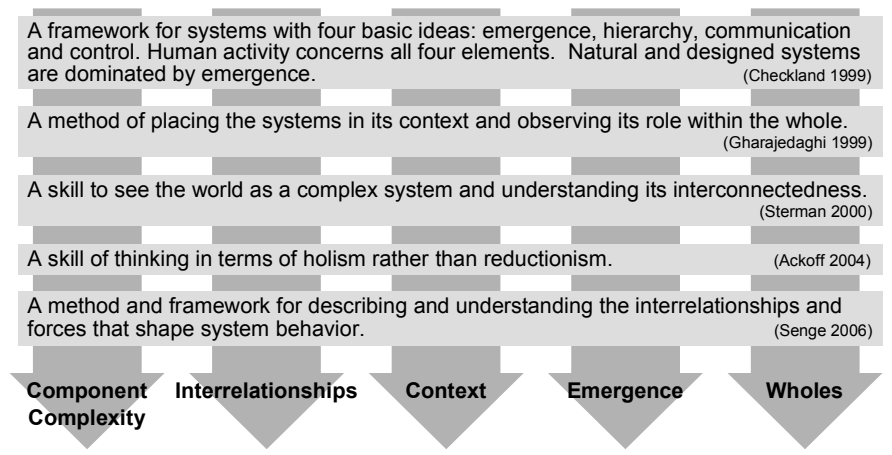

Fig. 1: Common definitions of systems thinking from the system dynamics community $[10,11,12,13$, and 14$]$

The second definition in Figure 1, "a method of placing the system in its context and observing its role within the whole," explicitly states a reliance on observation as part of systems thinking [12]. When engineers refer to systems thinking, the themes of wholism, emergence, and understanding patterns of behavior still apply, but do so in the context of realizing new systems, systems that have not yet been observed. As such, the engineering definitions of systems thinking place a greater role on interactions and interfaces because these contribute to emergence.

Referred to in [15], recent research has focused on differentiating and defining what is sometimes referred to as engineering systems thinking. These efforts recognize the social and technical components of engineering systems thinking $[9,16]$. One study utilized over 200 interviews with practicing systems engineers to develop the following definition of systems thinking as "utilizing modal elements to consider the componential, relational, contextual, and dynamic elements of the system of interest [9]." In other works, system thinkers use a variety of tools, methods, thinking styles, models and processes to enable consideration of the context, interrelationships, and dynamics of a system and its elements.

The benefits of systems thinking are associated with problem solving [2]. These skills include the ability to understand dynamic systems behavior, identify feedback processes, explain pattern of system behavior, and the ability to influence that behavior $[17,18]$. Such skills are necessary to understand the limitations of systems models, interpret and influence non-linear processes, and recognize when time delays between systems inputs and outputs [18].

Effective systems thinkers require familiarity with the problem, its base of knowledge, and should be able to leverage both quantitative and qualitative data towards a solution [18]. This dual emphasis on technical and social intelligences enables systems thinkers to more effectively mobilize, organize, and coordination resources (human, financial and physical) towards the completion of systems design [19]. The development of systems thinking within individuals is dependent on experiential learning, specific individual traits (e.g. curiosity and tolerance for uncertainty) and an environment that values and supports systems thinking skill development [9].

\section{HIGHER ORDER SYSTEMS THINKING}

\section{A. Motivation}

The dual pressures of engineering demographics and increasing complex technology motivate understanding higher-level systems thinking. The first step along this process is to expand the current understanding from the individual level to the team level.

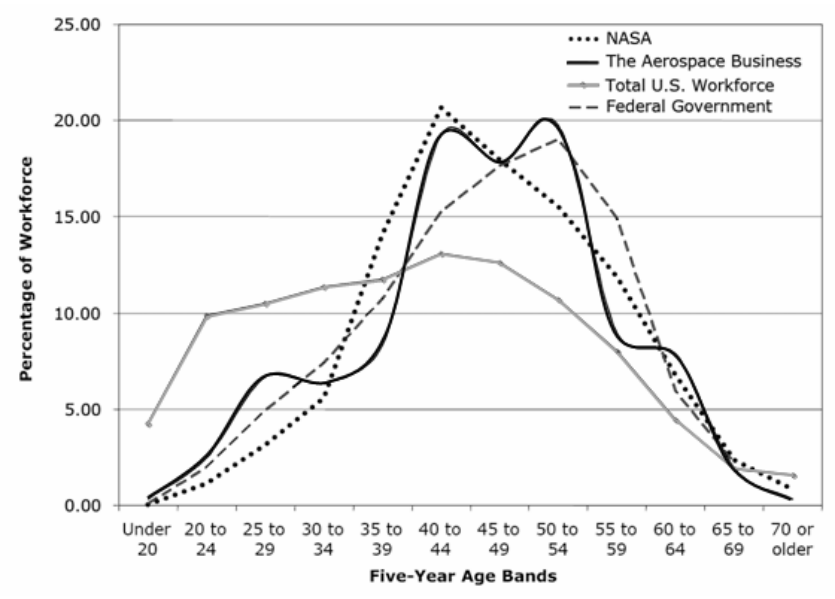

Fig. 2: Demographically, the aerospace workforce is much older than the US workforce as a whole and can expect $25 \%$ of the current workforce to retire by $2010[20,21]$

As engineers in this nation continue to retire faster than their replacements graduate, the engineering workforce is aging. The bulk of systems design experience is set to retire within the next 5-10 years [20]. Given the large fraction of tacit system-level design knowledge, $70 \%+[1]$, this mass 
retirement threatens key knowledge resources within the industry. Figure 2 shows the demographics of the aerospace industry, which are similar to most engineering fields. Program cycles and political cycles have left their imprint on the workforce. The majority of aerospace workers entered the industry during the Apollo era or the Cold War. The low numbers in the 20-40 demographic correspond with the end of the Cold War and reduced defense spending [21]. As the $50+$ age demographic retires, the remaining workforce is small, young, and relatively inexperienced.

Reductions in defense spending and increased systems complexity have resulted in fewer program starts and longer program lifecycles. Figure 3 shows the expected number of manned fighter programs starts over the course of a 40-year career by decade of graduation. An engineer entering the workforce in 1950 would have had the opportunity to work on nearly 50 manned fighter programs [22]. That same engineer entering the workforce today might only see two or three such programs. The same pattern is repeated for spacecraft and commercial aircraft [23, 24]. Fewer opportunities to go through the development cycle mean fewer opportunities to gain the experience shown to enable good systems thinking.

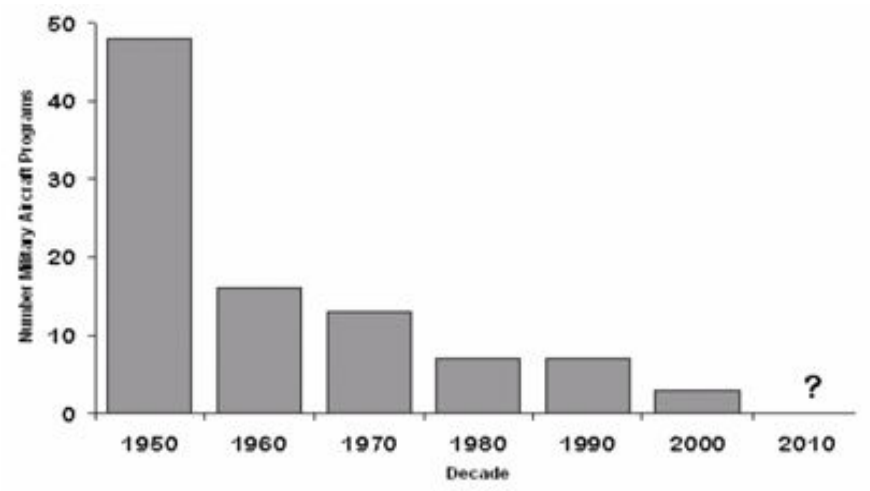

Fig. 3: Manned-fighter program starts over the course of a 40-year career by decade of entrance to workforce [22]

Focusing on systems thinking at the team level offers a potential solution in that emphasizing systems thinking within teams creates a supportive environment that values systems thinking and sharing design knowledge. It is hypothesized that successful systems thinking teams have a learning culture that will transmit design knowledge from the more experienced engineers near retirement to younger and lesser experienced engineers. Not only will these younger engineers be engaging in systems thinking within their teams, they will be learning design knowledge that will compensate for the reduced number of experiential learning opportunities over their careers. In addition to the workforce development advantages of team-level systems thinking, leveraging systems thinking at the team level will all the team as a cohesive unit to leverage a broader range of experiences and knowledge when faced with a design problem.

Systems of systems are higher than systems on the complexity hierarchy and therefore require systems thinking at a higher level. Intra-organizational units would be required to access the tacit knowledge of a system of system's constituent systems and effectively apply systems thinking.

\section{B. Definition}

Team-level systems thinking, or collaborative systems thinking, is "an emergent behavior of teams resulting from the interactions of team members and utilizing a variety of thinking styles, design processes, tools and communication media to consider the system, its components, interrelationships, context, and dynamics toward executing systems design [25]."

The definition of collaborative systems thinking builds upon that of [9] and incorporates the five key systems thinking themes shown in Figure 1. What differentiates collaborative systems thinking from individual systems thinking is the concept of team thinking, or the group processing of information through recall and interpretation [26]. Within engineering, thinking is the purposeful, reasoned and goal-directed action towards solving a problem. The elements of thinking in this context are decision making, problem exploration (creativity), judgment of alternatives, and ultimately problem solving [26]. The process begins with an ill-defined problem and uses recalled knowledge (memory) and other inputs towards solving the problems.

Collaborative systems thinking uses social interaction and information exchanges (conversations, sketches, equations and models) as the basis for a team conceptualizing a system and making systematic decisions. Because teams have multiple people contributing their knowledge and interpretation of that knowledge, teams are deemed better at making decisions, especially in safety critical situations. However, team skills are more difficult to develop as they must be practiced as a team [13]. Team thinking emerges from the intersection of individual team members' thinking, their behaviors and team processes, enabling a team to deliver more value than a group of individuals [27]. Throughout the process of problem solving, teams use communication to stimulate their thinking and handle uncertainty inherent in design [13]. The ways in which team communicate are determined in large part by team norms and processes [28], motivating a research concentration on culture and technical process as enablers for collaborative systems thinking.

\section{IV.APPROACH AND INITIAL RESULTS}

\section{Research Focus}

The goals of this research are to identify the mechanisms enabling collaborative systems thinking and generalize the traits of collaborative systems thinking teams. To focus the research, standard technical process and organizational culture were chosen as the foci of team investigation.

Because the questions entail observing and describing real-world phenomena, grounded theory methods are used to 
combine qualitative and quantitative data toward identifying the enablers and barriers of collaborative systems thinking.

Grounded theory research is characterized by concurrent and systematic data collection, analysis, and theory development [29, 30]. Grounded theory development involves the systematic collection of data from several sources including, but not limited to, surveys, interviews, focus groups, field observations and primary documents. From these sources, concepts and categories are identified and linked to form patterns. These patterns form the theory [30]. The formal coding process used in grounded theory builds up categories and constructs, selecting data from several sources and identifying conflicts and holes in data. These contradictions and holes then drive further data gathering and subsequent analysis. Because grounded theory research utilizes a systematic process to collect and analyze data, it leads to a more accurate process of discovery.

The goal of theory, of course, is to provide explanatory power in a specific, practical situation. In researching systems engineering, the goal is to explain the process by which engineers execute systems engineering; to predict and explain which behaviors and activities are helpful or harmful. Grounded theory provides a rigorous framework within which to collect and analyze data and avoid the pitfalls of revelation and intuition which threaten to relegate systems engineering to a philosophy rather than a science [31].

Grounded theory provides the data analysis structure within a three-phase research plan including a survey of applicable literature, pilot interviews to test initial ideas, and a field research phase utilizing case studies to provide the actual data for analysis. Phases 1 and 2 are important in gauging what are the correct questions to ask and metrics to use.

\section{Phase 1: Survey of Literature}

The first phase of research is a literature review. By drawing upon diverse fields such as systems dynamics, systems engineering, team cognition, psychology, organizational culture and workforce development, a wide range of potential influences were considered. The elements of team, process, and culture were chosen on the basis of available literature and offer an extension of the key enablers to individual systems thinking, as determined by [32]: individual characteristics, experiential learning, and supportive environment.

The cultural framework used is based on [33] and includes visible structure and process, strategic goals, and shared, underlying beliefs. This framework is supplemented by engineering cultural archetypes typifying many of the behavioral and underlying beliefs permeating engineering teams and organizations. These archetypes include the technophile, the expert and the non-communicator [34]. Each archetype provides insight into the productive and counterproductive tendencies of engineers and is used to formulate questions used in Phase 3.

\section{Organizational Culture \\ Standardized Technical Process}

\begin{tabular}{|l|r|}
\hline Team norms & $\begin{array}{r}\text { Documented tasks } \\
\text { and methods }\end{array}$ \\
\hline $\begin{array}{l}\text { Espoused } \\
\text { beliefs }\end{array}$ & $\begin{array}{r}\text { Vision } \\
\text { statements }\end{array}$ \\
\hline $\begin{array}{l}\text { Underlying } \\
\text { assumptions }\end{array}$ & $\begin{array}{r}\text { Strategy for } \\
\text { standardization }\end{array}$ \\
\hline $\begin{array}{l}\text { Social } \\
\text { networks }\end{array}$ & $\begin{array}{r}\text { Process flow } \\
\text { maps, org-charts }\end{array}$ \\
\hline
\end{tabular}

Fig. 4: Framework for evaluating culture and technical process within team context

The process literature consulted emphasized systems engineering processes: concept exploration, program definition, engineering and manufacturing, and production and field support [8]. Other literature took a more basic approach to process, breaking common practice into normative and natural camps [4]. The differentiator between normative and natural design processes is in the order of the steps during early design. The natural design process proceeds almost immediately to evaluation of a concept, whereas the normative process engages in-depth analysis of the problem and solution space before transitioning to concept evaluation [4]. The natural process is reliant on convergent thinking, where as the normative process engages both divergent and convergent thinking styles. The normative process has been shown to better handle design complexity [4], and is therefore of greater interest when considering collaborative systems thinking. Teamwork literature was also consulted for input on the role of process in team norm formation. This literature reinforced the pattern of culture and process interacting to form a team identity and enable the sharing of ideas [28]. Because this research exists within the aerospace domain, the industry emphasis on process standardization and maturity were also considered as important elements.

The outcome of the literature search was a framework relating the key variable of culture and technical process, shown in Figure 4. By identifying and linking the key components of culture and process, leverage points were identified that drove interview and survey design. This framework is discussed in more detail in [35].

Phase 1 also yielded information on team-based thinking, the role of creativity in systems thinking, ways in which personality types dictate team norms and thinking styles. 
Team thinking includes the ability to tolerate uncertainty, see the 'big picture,' think and take action as a team, and to communicate using the multiple languages of design (e.g. sketching, equations, models, etc.) [36]. The themes of 'big picture' thinking and tolerating uncertainty draw clear parallels between design thinking and systems thinking.

Creativity was another recurring theme within the literature. Creativity is linked to divergent thinking and problem solving and is supported by well-defined sets of team norms and beliefs [37]. Established processes, such as brainstorming, also exist to promote creativity within teams. Because engineering cultural norms gravitate towards convergent thinking styles and a tendency to minimize interpersonal communication, processes and norms that promote creativity are likely to enable collaborative systems thinking. Further literature on the role of personality types in team performance indicated little research had looked at tailoring process to team personality type [38] despite the predominance of specific personality traits among engineers.

While a great deal of research was found on the topic of engineering team thinking and problem solving, all of this literature focused on student project teams. No similar studies found have undertaken such observation within practicing engineering teams because of the logistics entailed.

\section{E. Phase 2: Pilot Interviews}

Phase 2 utilized pilot interviews to validate the initial concepts and directions determined by the literature review. Twelve semi-structured pilot interviews and dozens of information conversations informed this stage. Because the goal was idea validation, no coding or numerical analysis was applied to the results of pilot interviews. Rather, compelling ideas were further explored and additional literature sources were added to Phase 1 of this research. The pilot interviews did show an overwhelming consensus that culture and process are well chosen venues through which to explore collaborative systems thinking. A complete summary of plot interview results is provided in [15].

Questions asked focused on the differences between individual systems thinking and collaborative systems thinking and on providing illustrative examples of collaborative systems thinking teams and the traits that enabled their success.

Identified differences between individual and collaborative systems thinking include the need for team communication and the concept that while individuals contribute to a project, teams are responsible for delivering completed projects. These themes are included in the definition of collaborative systems thinking through the qualifications of "interactions of team members" and "towards executing system design."

The illustrative examples of collaborative systems thinking teams had the common themes of being productcentric (in contrast to functional or process centric) and exhibiting good team awareness. Team awareness includes not only being aware of what other team members are doing, but also of their past experiences and working styles. This theme was often manifested in terms of good project management and teams composed of individuals with similar working styles.

\section{F. Phase 3: Case Studies}

Phase 3 combines the preparation and vetting of phases 1 and 2 into a field study of aerospace engineering teams. Case studies are the primary data collection method. Flexible and effective, case studies are well suited for exploratory research and enable the gathering of many different types of data [39]. Case studies are also helpful in establishing external validity of the collected data and for ensuring obtained results are sufficiently generalizable [39].

Case studies utilize structured methods (e.g. surveys and structured interviews) and unstructured methods (e.g. observation and semi-structured or unstructured interviews). Structured data collection methods are well suited to collecting team demographics and process metrics. Unstructured methods are better suited for measures of culture and perceptions of team collaborative systems thinking capability. Wherever possible, validated questions and metrics from relevant past research are being used to reduce construct and discriminant validity concern.

Table 1: Criteria for case study selection

\begin{tabular}{|l|l|l|l|l|l|}
\hline \multicolumn{3}{|l|}{$\begin{array}{l}\text { Independent } \\
\text { Variables }\end{array}$} & $\begin{array}{l}\text { Dependent } \\
\text { Variable }\end{array}$ \\
\hline $\begin{array}{l}\text { Industry } \\
\text { Sector }\end{array}$ & Customer & $\begin{array}{l}\text { Program } \\
\text { Lifecycle }\end{array}$ & $\begin{array}{l}\text { Process } \\
\text { Maturity }\end{array}$ & $\begin{array}{l}\text { Team } \\
\text { Size }\end{array}$ & $\begin{array}{l}\text { Collaborative } \\
\text { Systems } \\
\text { Thinking }\end{array}$ \\
\hline $\begin{array}{l}\text { Aircraft } \\
\text { (Hardware) }\end{array}$ & US Gov. & $\begin{array}{l}\text { Conceptual } \\
\text { Design }\end{array}$ & High & $<10$ & Strong \\
\hline $\begin{array}{l}\text { Spacecraft } \\
\text { (Hardware) }\end{array}$ & Commercial & $\begin{array}{l}\text { Detail } \\
\text { Design }\end{array}$ & Low & $>10$ & Weak \\
\hline Software & Private & & & & \\
\hline
\end{tabular}

To ensure a representative sample, selection criteria have been established for case studies, as shown in Table 1. These criteria guide the selection process to ensure a wide sample, and therefore generalizable results, is obtained. Industry sector assures that a variety of technologies and industry cultures are observed. Likewise the systems customer is an indicator of culture, with government programs being subject to different time reporting procedures and technical process requirements. Selecting teams in the conceptual or detail design phase controls for variability in the frequency and type of team communication that occurs at different stages in the program lifecycle. The early stages of design were chosen to observe teams engaging in rich communication. While standard process maturity models award ratings from 1-5, the process maturity criteria is binary and meant to indicate whether a company has thorough and well adhered-to processes in place. The final criterion, team size, is for 
calibration of communication data. Smaller teams are likely to have denser communications and greater team awareness compared to larger teams.

A single case study is designed to span two or three days in addition to advanced preparations. Basic information relative to the selection criteria is collected in advance of the case study. Advance information includes the contact's assessment of the team's collaborative systems thinking. The first day is dedicated to introducing the research and administering the survey to the entire engineering team. The survey takes approximately 30 minutes and focuses on team demographics, measures of process adherence, and perceptions of process effectiveness and environment. Several survey questions are geared at measuring elements of design thinking, effective communication, and a supportive environment with a goal of confirming or contesting the initial collaborative systems thinking assessment. Because no validated measures exist for systems thinking, let along collaborative systems thinking, self reporting, related indicators and third party assessments will be used to gage the collaborative systems thinking capability of a team. The second day (and third day if necessary) is for follow-up interviews with pre-selected team members and team supervisors. Semi-structured interviews are used to engage the interviewee in a discussion about team and organizational culture. The interviews will collect primarily qualitative data to be analyzed using coding methods. Between four and six people from each team will be included in the follow-up interviews.

Approximately 20 case studies will be completed to sample the 56 possible combinations of selection criteria. Because this is exploratory research, the goal is to sample until saturation, or when new observations do not add new information to the understanding of collaborative systems thinking. As of the publication of this paper, case studies are under way with a target completion date of Fall 2008.

\section{V.FUTURE WORK}

\section{G. Continuing Team-Level Research}

The research described above is ongoing and should be completed by early 2009 . At that point complete results will be published and shared with all participating organizations. Because the nature of the research is exploratory, the results will be descriptive. That is, the resulting theory of collaborative systems thinking will explain the observed circumstances.

For the theory to be prescriptive, additional research will be required. Future research at the team level should seek to apply the resulting theory to case studies both within and outside of the aerospace industry. Hypothesis testing will establish cause and effect relationships for informing corporate interventions aimed at developing collaborative systems thinking. Including case studies outside of the aerospace industry will render the results more generalizable.
Future research should also consider factors beyond culture and technical process. The physical work environment may play a large role in collaborative systems thinking. For instance, collocated teams may be more likely to engage in collaborative systems thinking because they are able to communicate on a tactile level. Visualization aids like black boards and physical models may also be factors. The frequency with which team members work together on different projects might also play a role as individuals who work together more frequently are more likely to be aware of each other's past experiences and work styles.

\section{H. Application to Systems of Systems}

Application of this research to systems of systems is a natural extension. However, whereas a systems design team comes from one culture and set of technical processes, system of systems are meld together multiple organizational culture and technical process sets. With this comes issues of trust with regards to protecting proprietary data and mismatches in cultural expectation.

Results from this research may inform the core set of values and information sharing processes to establish good communication within system of systems management. While the technical component of systems of systems is important, the social component is even more so because systems of systems exist only through the mutual agreement of individual systems owners.

Because system of systems management is a distributed and collaborative activity, many of the lessons learned in [40] are likely enablers of system of systems level systems thinking. These include the establishment of trust, investments in up-front planning and clear definitions of decision making responsibilities.

\section{CONCLUSIONS}

Literature and initial results indicate that organizational culture and technical process are important factors in leveraging team-level systems thinking. As part of an overall research program of systems engineering in the enterprise [15], this research is providing grounded empirical knowledge toward enabling a science of systems engineering.

\section{ACKNOWLEDGMENT}

The authors gratefully acknowledge the funding for this research provided through the Lean Advancement Initiative (LAI, http://lean.mit.edu) and the MIT Systems Engineering Advancement Research Initiative (SEAri, http://seari.mit.edu).

\section{REFERENCES}

[1] Q. Dong, Representing Information Flow and Knowledge Management in Product Design Using Design Structure Matrix, Master's thesis, Massachusetts Institute of Technology, Cambridge, MA, 1999. 
[2] P. Jansma and R. Jones, "Advancing the Practice of Systems Engineering at JPL", IEEE Aerospace 2006, Big Sky, MT, March 2006.

[3] C. Sanford, "A Theory and Practice System of 'Systems Thinking", 3rd International Conference on System Thinking in Management, Philadelphia, PA, May, 2004

[4] J. Stempfle and P. Badke-Schaub, "Thinking in Design Teams: An analysis of team communication", Design Studies, Vol. 23, No. 1, pp 473-496, 2002.

[5] D. Dougherty, "Understanding New Markets for New Products", Strategic Management Journal, Special Issue: Corporate Entrepreneurship, pp 59-78, 1990.

[6] S. Popper, S. Bankes, R. Callaway and D. DeLaurentis, System-ofSystems Symposium: Report on a Summer Conversation, July 21-22, 2004, Potomac Institute for Policy Studies, Arlington, VA.

[7] M.W. Maier, "Architecting Principles for System of Systems," Systems Engineering, Vol. 1, No. 4, pp. 267-284, 1998.

[8] J. Martin, Systems Engineering Guidebook, CRC Press, Boca Raton, FL, 1997.

[9] H. Davidz, Enabling Systems Thinking to Accelerate the Development of Senior Systems Engineers, $\mathrm{PhD}$ thesis, Massachusetts Institute of Technology, Cambridge, Massachusetts, 2006.

[10] R. Ackoff, "Transforming the Systems Movement", Opening Speech a 3rd International Conference on Systems Thinking in Management, Philadelphia, PA, May, 2004.

[11] P. Checkland, Systems Thinking, Systems Practice, Soft Systems Methodology: A 30-year retrospective, John Wiley and Sons, West Sussex, England, 1999

[12] J. Gharajedaghi, Systems Thinking: Managing chaos and complexity, Butterworth-Heinemann, Burlington, MA, 1999.

[13] P. Senge, The Fifth Discipline, Doubleday, New York, NY, 2006.

[14] J. Sterman, Business Dynamics: Systems thinking and modeling for a complex world, McGraw-Hill, New York, NY, 2000.

[15] D.H. Rhodes, C. T. Lamb and D.J. Nightingale, "Empirical Research on System Thinking and Practice in the Engineering Enterprise", IEEE International Systems Conference, Montreal, Canada, April, 2008.

[16] M. Frank, "Engineering Systems Thinking and Systems Thinking", Systems Engineering, Vol. 3, No. 3, pp 163-168, 2000.

[17] B. Richmond, "Systems Thinking: Critical thinking skills for the 1990s and beyond", System Dynamics Review, Vol. 9, No. 2, pp 113-133, 1993.

[18] L. Sweeney and J. Sterman. "Bathtub Dynamics: Initial results of a systems thinking inventory", System Dynamics Review, Vol. 16, No. 4, pp 249-294, 2000

[19] S. Beder, "Beyond Technicalities: Expanding engineering thinking," Journal of Professional Issues in Engineering Education and Practice, Vol. 125, No 1, pp 12-18, January 1999.

[20] N. Augustine and The Committee on Prospering in the Global Economy of the 21st Century, "Rising Above the Gathering Storm: Energizing and employing America for a brighter economic future", Technical report, National Academies, 2006

[21] D. Black, D. Hastings and the Committee on Meeting the Workforce Needs for the National Vision for Space Exploration, "Issues Affecting the Future of the U.S. Space Science and Engineering Workforce: Interim report", Technical report, National Academies, 2006.

[22] E. Murman et.al., Lean Enterprise Value: Insights from MIT's Lean Aerospace Initiative, Palgrave, New York, NY, 2002.

[23] Boeing Commercial Airplanes, http://www.boeing.com/commercial Accessed May 2007.

[24] V. Neal, C. Lewis and F. Winter, Spaceflight, Macmillan, New York, NY, 1995.

[25] C.T. Lamb and D.H. Rhodes, "Collaborative Systems Thinking: Towards and understanding of team-level systems thinking", 6th Conference on Systems Engineering Research, Redondo Beach, CA, April 2008.

[26] A. Akgun, G. Lynn and R. Reilly, "Multi-Dimensionality of Learning in New Product Development Teams", Journal of Innovation Management, Vol. 5, No. 2, pp 57-72, 2005.

[27] N. Cooke et.al., "Advances in Measuring Team Cognition", E. Salas and S. Fiore, editors, Team Cognition, chapter 5, pp 83-106, American Psychological Association, Washington, DC, 2004
[28] J.R. Hackman, Leading Teams, Harvard Business School Press Boston, MA, 2002.

[29] B. Glaser and A. Strauss, The Discovery of Grounded Theory, Aldine Publishing Company, Chicago, 1967.

[30] A. Strauss and J. Corbin, Basics of Qualitative Research, Sage Publications, Thousand Oaks, 1998.

[31] R. Valerdi and H. Davidz, "Empirical Research in Systems Engineering: Challenges and opportunities of a new frontier", Technical Proceeding of 5th CSER Conference on Systems Engineering Research, Hoboken, NJ, March 2007.

[32] H. Davidz, D. Nightingale, and D. Rhodes, "Enablers and Barriers to Systems Thinking Development: Results of a Qualitative and Quantitative Study," 3rd Conference on Systems Engineering Research, Hoboken, NJ, March 2005

[33] E. Schein, Organizational Culture and Leadership, Jossey-Bass, San Francisco, 2004.

[34] P. Leonardi, The Mythos of Engineering Culture: A study of communicative performance and interaction, Master's thesis, University of Colorado, Boulder, CO, June 2003.

[35] C.T. Lamb and D.H. Rhodes, "Standardized Process as a Tool for Higher Level Systems Thinking," INCOSE International Symposium 2007, San Diego, CA, June 2007.

[36] C. Dym et.al., Engineering Design Thinking, Teaching, and Learning. Journal of Engineering Education, Vol. 94, No. 1, pp 103-120, 2005.

[37] T. Varvel et.al., "Team Effectiveness and Individual Myers-Briggs Personality Dimensions", Journal of Management in Engineering, Vol. 20, No. 4, pp 141-146, October 2004.

[38] A. Volger, "The Munich Model: Creating an environment for space architecture development", AIAA Space Architecture Symposium, Houston, TX, October 2002.

[39] R. Yin, Case Study Research Design and Methods, Sage Publications, Sage, CA, 3rd edition, 2003.

[40] D. Utter, Collaborative Distributed Systems Engineering, Master of Science Thesis, Engineering Systems Division, MIT, January 2007. 